\title{
Left ventricular diastolic dysfunction is associated with cerebral infarction in young hypertensive patients: A retrospective case-control study
}

\author{
HUI-JUN WEN and XIAO-YONG WANG \\ Department of Neurology, Baoji Municipal Central Hospital, Baoji, Shaanxi 721008, P.R. China
}

Received August 24, 2019; Accepted January 23, 2020

DOI: $10.3892 /$ etm.2020.9189

\begin{abstract}
Studies have indicated that hypertension is associated with the occurrence of acute cerebral infarction (CI) in young patients (18-45 years). However, the association between CI and left ventricular diastolic (LVD) dysfunction in young patients with hypertension has rarely been reported. The purpose of the present study was to investigate the association between LVD dysfunction and acute CI in young patients with hypertension. A total of 92 patients with acute CI who had hypertension were selected as the study group (CI group) and 98 young patients with only hypertension were selected as the control group (non-CI group). Blood pressure measurements, LVD functional assessment and cerebral MRI were performed. The $\chi^{2}$ test was used to compare the left ventricular diastolic function between the CI and non-CI groups. The results indicated that LVD function of young patients was associated with hypertension and there was a correlation between the decrease in LVD function and the occurrence of acute CI in young patients with hypertension. The incidence of acute CI was higher in patients with decreased LVD function than in those with normal LVD function. In conclusion, hypertension in the young is associated with decreased LVD function and is a risk factor for diastolic dysfunction of the left ventricle. LVD function may be an independent predictor of acute CI in young patients with hypertension and should be considered by clinicians. By predicting the risk of acute $\mathrm{CI}$ in young patients with hypertension, LVD testing may aid in the primary prevention of CI or guide early treatment.
\end{abstract}

Correspondence to: Dr Xiao-Yong Wang, Department of Neurology, Baoji Municipal Central Hospital, 8 Jiangtan Road, Baoji, Shaanxi 721008, P.R. China

E-mail: whjsq2008@163.com

Key words: hypertension, left ventricular diastolic dysfunction, infarction, young patients, risk factors, cerebral infarction, cardiac morphology

\section{Introduction}

It has been speculated that the occurrence of cerebral infarction (CI) caused by most heart diseases may be associated with the reduction of cardiac output (1). Hooghiemstra et al (2) think that the hemodynamic balance of the 'heart-brain axis' is the key factor in maintaining brain structure and any node problem may lead to cerebrovascular disease in the vascular pathway from the heart to the brain. Recent studies have indicated that left ventricular diastolic (LVD) dysfunction may occur in hypertensive patients when their heart structure is still normal (3). Left atrium, left ventricular configuration and functional changes have been associated with acute $\mathrm{CI}$ in patients with hypertension or coronary heart disease (4). According to Nam et al (5), LVD function is an independent risk factor for cerebral small vessel disease. There has even been an increase in the incidence, prevalence and duration of hypertension along with a significant rise in the incidence of cerebrovascular atherosclerosis and small vessel disease (6), whose cumulative effects cause vascular endothelial damage and ultimately lead to cerebral vascular occlusion.

A study by Shimizu et al (7) determined a positive correlation between LVD dysfunction and cerebral white matter lesions in elderly patients. A multicenter prospective cohort study indicated that the left atrial diameter index was significantly associated with the risk of stroke recurrence (8). Youth hypertension is defined as hypertension occurring in patients aged between 18 and 45 years. Importantly, the proportion of young individuals among patients with stroke is increasing and the risk of disease increases even further with age (9). It should be noted that lifestyle changes, early intervention in hypertension, exercise, regular examination and other precautions are all important for the prevention of CI in young individuals (10). In fact, a combination of several effective approaches is the most effective way to restrict the occurrence and development of CI (11). Although studies have suggested that the decline of LVD function is a transfer from a physiological to a pathological process and that an early manifestation of hypertension in heart damage is the reduction of diastolic function (12), there have been no clinical reports on the association between LVD dysfunction and CI among young patients with hypertension, to the best of our knowledge. The present study aimed to identify the association between LVD and acute CI in young patients with hypertension. 


\section{Materials and methods}

Patients. The present study was approved by the Ethics Committee of the Medical Ethics Committee of Baoji Central Hospital (Baoji, China). All procedures performed in studies involving human participants were in accordance with the ethical standards of the 1964 Helsinki Declaration and its later amendments. Written informed consent for data collection was obtained. A retrospective case-control study was performed. A total of 92 patients were diagnosed as hypertensive with acute $\mathrm{CI}$ (CI group), comprising 55 males and 37 females aged $35-44$ years (mean age, $35.26 \pm 8.36$ years) who were treated or hospitalized at the Department of Neurology at Baoji Central Hospital. A total of 98 patients were diagnosed with hypertension (non-CI group), comprising 65 males and 33 females aged 36-45 years (mean age, $36.59 \pm 9.07$ years); the patients were retrospectively selected based on their general medical examination records obtained between January 2014 and February 2018. Blood pressure measurements, LVD functional assessment and brain MRI were performed in all patients. According to the results of echocardiography, patients were divided into the LVD function reduction group and the normal LVD function group. A flow chart of the scheme of the present study is provided in Fig. 1.

Major equipment. The following pieces of equipment were employed: i) Echocardiography detector: Siemens s2000 echocardiograph instrument (Siemens AG); ii) Magnetic resonance diffusion weighted imaging: SIEMENS ESSENZA 1.5T magnetic resonance scanner (Siemens AG); iii) Biochemical analyzer: AU2700 biochemical analyzer (Olympus).

Determination of LVD function. According to a system described in the international literature (13), the degree of LVD dysfunction was classified into grades numbered 0-3 with a higher number indicating greater severity; grades 1 and above indicated clinical diastolic dysfunction. The left ventricular isovolumic relaxation time (IVRT) was measured by quantitative tissue velocity imaging (QTVI) on tissue Doppler echocardiography (normal values: Age of $<40$ years, $69 \pm 12 \mathrm{msec}$; age of $>40$ years, $76 \pm 13 \mathrm{msec}$ ). The peak velocity between the early filling and late filling of the left atrioventricular (AV) plane motion (Ea/Aa) (normal value: $>1$ ) and the left atrial annulus systolic velocity (A'value) (normal value: $4.89 \pm 0.67 \mathrm{~cm} / \mathrm{sec}$ ) are used to assess LVD function in patients. The higher the IVRT and A'value or the lower the Ea/Aa, the more severe is the LVD function damage (14-18).

Diagnosis of CI. The inclusion criteria were as follows: i) Stroke onset had occurred within the past 7 days; ii) the case conformed to the diagnostic criteria of the 2014 Chinese Acute Ischemic Stroke Diagnosis and Treatment Guidelines (19), in that the symptoms and signs of neurological deficits were well defined and persisted for $24 \mathrm{~h}$ without any significant relief and the lesion was identified by cranial MRI; iii) the patient consented to participate in the study and was able to cooperate with the examination; iv) the patient had primary CI. The exclusion criteria were as follows: i) The patient was $<18$ years or $>45$ years of age at the time of the study; ii) the stroke did not conform to the diagnostic criteria in the 2014 Chinese Acute Ischemic Stroke Diagnosis and Treatment Guidelines; iii) the clinical data were incomplete; iv) due to stroke, a patient suffering from aphasia, mental disorders or dementia was not able to provide a complete medical history and the family members were not able to provide the information either; $v$ ) the patient had hemorrhagic stroke; vi) the patient had cardiogenic CI or CI due to autoimmune or blood system disorders; vii) the stroke was accompanied by severe heart, liver or kidney disease; viii) the patient was using hormones, immunosuppressants or non-steroidal anti-inflammatory drugs prior to and after the onset of CI; ix) the patient had CI due to the onset of arteritis.

Lipid abnormalities. Blood was collected from the elbow vein of the subject with a fasting time of $>8 \mathrm{~h}$ and the serum was isolated within $2 \mathrm{~h}$. Blood lipids were determined with the Cobas- 8000 automatic biochemical analyzer (Roche). In the present study, lipid abnormality refers to abnormalities in the quantity and quality of lipids in plasma. The 2013 American College of Cardiology/American Heart Association American Adult Guidelines for the Reduction of Atherosclerosis Lipids recommend low-density lipoprotein cholesterol (LDL-C) $<1.8 \mathrm{mmol} / \mathrm{l}(20)$.

Laboratory biochemical indicators. Fasting blood glucose and LDL-C were measured using an Olympus AU2700 biochemical analyzer (Olympus Corp.).

Statistical analysis. The statistical software SPSS 20.0 (IBM Corp.) was used for statistical analysis. Clinical data were manually collected, and statistical software was used to organize and analyze data to draw meaningful conclusions based on the experimental results. The Wilcoxon rank-sum test was used in the analysis of count data. Values of measurement data are expressed as the mean \pm standard deviation. The $\chi^{2}$ test was used to compare blood pressure and LVD function between patients in the CI group and non-CI group. The $\chi^{2}$ test was also used to compare pre-infarction and post-infarction diastolic function in patients of the CI group.

\section{Results}

Comparison of baseline data between the two groups. Patients were excluded if they had lesions that may affect the blood pressure, including secondary hypertension, arrhythmia, coronary heart disease, valvular heart disease, anemia or hyperthyroidism. The factors of gender, age, blood lipids, blood glucose, smoking, alcohol consumption and family history of vascular disease exhibited no statistically significant difference between the CI group and the non-CI group ( $\mathrm{P}>0.05)$, meaning that the groups were comparable with respect to these factors (Table I). There was no significant difference in blood pressure between the two groups, meaning that they were comparable in this regard as well (Table II).

Grading comparison of left ventricular diastolic function in all patients included in the study. According to the left heart function grading standards reported in the international literature (3), the data of the present study indicated the following: Among the 190 patients, 18 (9.47\%) had grade 0 diastolic dysfunction (normal ventricular diastolic function) and 172 (90.53\%) had diastolic dysfunction. Among the patients with 


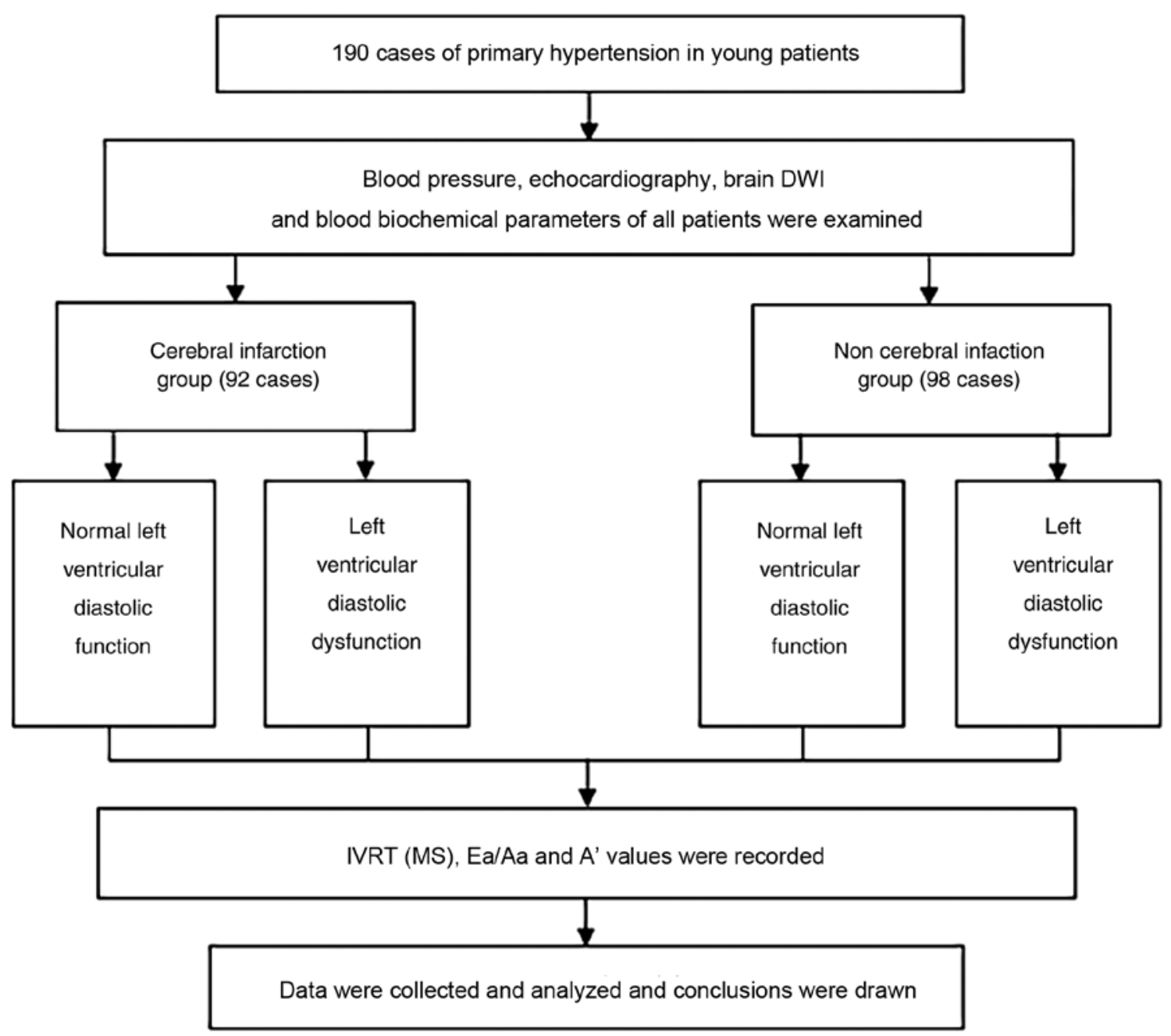

Figure 1. Flow chart of the study. A total of 190 patients with hypertension were enrolled and their blood pressure, cardiac function, brain DWI and blood biochemical indicators were determined. The CI group (92 cases) and non-CI group (98 cases) were divided according to their brain DWI results. The left ventricular diastolic function of 190 patients was measured. There were 4 cases with normal left ventricular diastolic function and 88 cases with abnormal left ventricular diastolic function in the CI group. In the non-CI group, there were 14 cases with normal left ventricular diastolic function and 84 cases with abnormal left ventricular diastolic function. The data were collected, statistical analysis was performed and final conclusions were drawn. DWI, diffusion-weighted imaging; CI, cerebral infarction; IVRT, isovolumic relaxation time; Ea/Aa, peak flow velocity of the left atrioventricular annulus at the early stage of filling/peak flow velocity of the left atrioventricular annulus at the late stage of filling; A'value, atrial tissue velocity of the left atrioventricular valve annulus.

diastolic dysfunction, 117 cases $(61.58 \%)$ had grade 1,47 cases (24.74\%) had grade 2 and 8 cases $(4.21 \%)$ had grade 3 diastolic dysfunction (Table III).

Comparison of left ventricular diastolic function before and after infarction in patients with cerebral infarction. The LVD function indexes of the CI group prior to infarction and after infarction were compared. The IVRT and A'values of the CI group after infarction were higher than those of prior to infarction, whereas the Ea/Aa value was lower. The difference was statistically significant $(\mathrm{P}<0.05$; Table IV).

Comparison of left ventricular diastolic function between the cerebral infarction group after infarction and the non-infarction group. The LVD function indexes of the CI group after infarction and the non-CI group were compared. The IVRT and A'values of the CI group after infarction were higher than those of the non-CI group, whereas the Ea/Aa value was lower. The difference was statistically significant $(\mathrm{P}<0.05$; Table $\mathrm{V}$ and Fig. 2).

\section{Discussion}

Young ischemic stroke (YIS) refers to ischemic stroke occurring in patients aged 45 or under and its incidence is increasing annually (14). The reason is probably its characteristics of early onset, early disability and high recurrence. At present, YIS accounts for an estimated 5-10\% of all cases of CI and the most common pathogenetic origin of the disease is atherosclerosis caused by hypertension (21). The pathogenic effect of hypertension has been indicated to be directly correlated with its severity and duration, which indicates a connection between hypertension and an increased risk of YIS (22).

Young patients pay insufficient attention to blood pressure and frequently remain untreated (23). This long-term elevation of peripheral blood pressure increases cardiac after-loading, while increased peripheral vasoconstriction tension and retention of sodium and water increase myocardial oxygen consumption, deteriorate myocardial compliance and augment ventricular end-diastolic pressure (24). As a result, cardiac preload increases and left ventricular hypertrophy occurs, 
Table I. Comparison of baseline data between the two groups.

\begin{tabular}{lcc}
\hline Group & CI (92) & Non-CI (98) \\
\hline Age (years) & $35.26 \pm 8.36$ & $36.59 \pm 9.07$ \\
Male sex & $55(28.95 \%)$ & $65(34.21 \%)$ \\
Dyslipidemia & $48(25.26 \%)$ & $42(22.11 \%)$ \\
Abnormal fasting blood glucose & $6(3.16 \%)$ & $4(2.11 \%)$ \\
Smoking & $36(18.95 \%)$ & $32(16.84 \%)$ \\
Alcohol consumption & $28(14.74 \%)$ & $23(12.11 \%)$ \\
Family history of vascular disease & $4(2.11 \%)$ & $3(1.58 \%)$ \\
\hline
\end{tabular}

Values are expressed as $\mathrm{n}$ or the mean \pm standard deviation. There were no significant inter-group differences. CI, cerebral infarction.

Table II. Comparison of hypertension between the two groups.

\begin{tabular}{lccc}
\hline Group & $\mathrm{N}$ & $\mathrm{SBP}(\mathrm{mmHg})$ & $\mathrm{DBP}(\mathrm{mmHg})$ \\
\hline CI group & 92 & $167.18 \pm 12.75$ & $103.36 \pm 7.03$ \\
Non-CI group & 98 & $159.26 \pm 15.92$ & $105.28 \pm 6.74$ \\
$\chi^{2}$ & & 25.436 & 16.125 \\
P-value & & 0.062 & 0.057
\end{tabular}

Values are expressed as the mean \pm standard deviation. SBP, systolic blood pressure; DBP, diastolic blood pressure.

Table III. Classification of left ventricular diastolic function in the patients of the present study $(n=190)$.

\begin{tabular}{lcc}
\hline Classification & Grade & $\mathrm{n}(\%)$ \\
\hline Normal diastolic function & 0 & $18(9.47)$ \\
Early diastolic dysfunction & 1 & $117(61.58)$ \\
False normalization & 2 & $47(24.74)$ \\
Restrictive filling disorder & 3 & $8(4.21)$ \\
\hline
\end{tabular}

whereas diastolic function is significantly reduced (25). Cerebral hypoperfusion, combined with intracranial arteriosclerosis and even occlusion caused by high blood pressure, eventually leads to CI (26). It has been indicated that an early onset of hypertension always triggers reduced LVD function (3). The decrease precedes a change in ventricular systolic function and leads to an altered cardiac configuration $(24,25)$. Cerrato et al (6) reported that heart problems accounted for $24 \%$ of the causes of YIS. Another study reported that acute CI may be associated with LVD dysfunction, which has an important role in the pathogenesis and prognosis of acute CI (26). In the present study, 172 patients with LVD dysfunction accounted for $90.53 \%$ of the 190 patients enrolled, which suggests that hypertension may cause LVD dysfunction in young patients. Diastolic function of grades I and II accounted for the largest proportion, indicating that the incidence of early diastolic dysfunction is high in young hypertensive patients. These results suggest a requirement to actively perform early
Table IV. Comparison of left ventricular diastolic function indexes prior to and after infarction in the cerebral infarction group $(n=92)$.

\begin{tabular}{lccc}
\hline Time-point & IVRT $(\mathrm{msec})$ & Ea/Aa & A'value $(\mathrm{cm} / \mathrm{sec})$ \\
\hline Prior to infarction & 81.37 & $1.15 \pm 0.12$ & $5.10 \pm 0.92$ \\
After infarction & $114.97 \pm 10.16$ & $0.79 \pm 0.11$ & $5.21 \pm 1.03$ \\
$\chi^{2}$ & 19.146 & 16.541 & 15.019 \\
P-value & 0.048 & 0.040 & 0.046 \\
\hline
\end{tabular}

Values are expressed as the mean \pm standard deviation. IVRT, isovolumic relaxation time; Ea/Aa, peak flow velocity of the left atrioventricular annulus at the early stage of filling/peak flow velocity of the left atrioventricular annulus at the late stage of filling; A'value, atrial tissue velocity of the left atrioventricular valve annulus.

Table V. Comparison of left ventricular diastolic function indexes between the two groups.

\begin{tabular}{lccc}
\hline Group & IVRT (msec) & Ea/Aa & A'value $(\mathrm{cm} / \mathrm{sec})$ \\
\hline CI group & $114.97 \pm 10.16$ & $0.79 \pm 0.11$ & $5.21 \pm 1.03$ \\
(post-infarction, 92) & & & \\
Non-CI group (98) & $72.86 \pm 8.75$ & $1.3 \pm 0.25$ & $5.02 \pm 0.82$ \\
$\chi^{2}$ & 23.124 & 17.026 & 14.351 \\
P-value & 0.039 & 0.037 & 0.042 \\
\hline
\end{tabular}

Values are expressed as the mean \pm standard deviation. IVRT, isovolumic relaxation time; Ea/Aa, peak flow velocity of the left atrioventricular annulus at the early stage of filling/peak flow velocity of the left atrioventricular annulus at the late stage of filling; A'value, atrial tissue velocity of the left atrioventricular valve annulus; CI, cerebral infarction.

diagnosis and early treatment to prevent the occurrence of acute cerebrovascular events, which is essentially consistent with the conclusions of previous studies.

The reason why LVD dysfunction induces abnormal peripheral hemodynamics followed by cerebral hypoperfusion has yet to be identified. First, we surmise that with decreasing diastolic function, the left ventricle cannot effectively expand, causing the returned blood volume and cerebral perfusion blood to decrease (27). Second, the decreased LVD function leads to a decrease in blood flow and friction between the endothelia of blood vessels, atherosclerosis is promoted, acute cerebrovascular occlusion is created and CI ultimately occurs (28). Early detection, accurate evaluation and timely prevention of LVD dysfunction are clinically significant for the prevention of $\mathrm{CI}$.

According to the American Society of Echocardiography guidelines (13), LVD function depends mainly on left ventricular flaccidity and left ventricular compliance. The pressure indexes measured by left cardiac catheterization, including left ventricular end-diastolic pressure, maximum left ventricular pressure drop rate and the left ventricular relaxation time constant are regarded as the gold standard for assessing LVD dysfunction. The guideline recommends the $\mathrm{Ea} / \mathrm{Aa}$ ratio from 

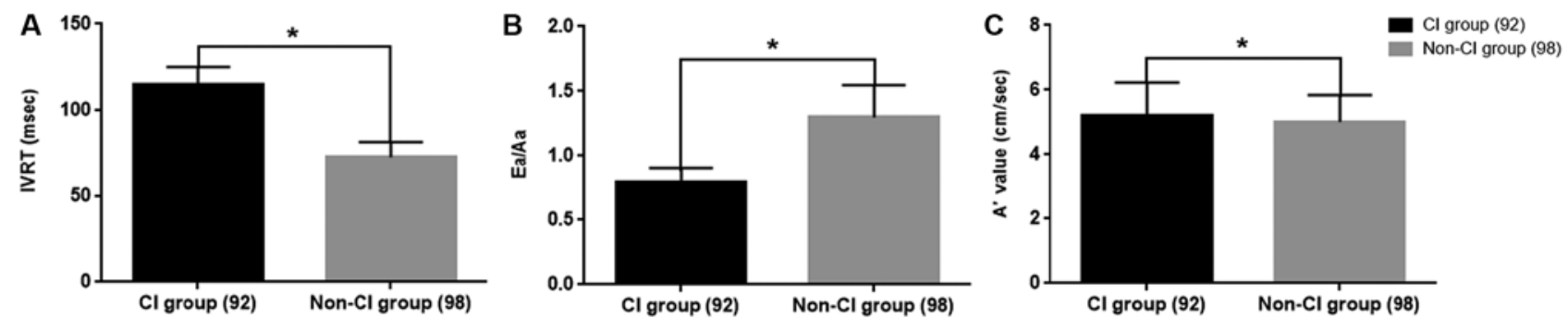

Figure 2.Left ventricular diastolic function between 92 patients with CI and 98 patients without CI and with hypertensionwere compared. The IVRT and A'value in the CI group were higher than those in the non-CI group (A and C), while the Ea/Aa in the CI group was lower than that in the non-CI group (B), and there were significant differences between the groups (" $\mathrm{P}<0.05)$. CI, cerebral infarction; IVRT, isovolumic relaxation time; Ea/Aa, peak flow velocity of the left atrioventricular annulus at the early stage of filling/peak flow velocity of the left atrioventricular annulus at the late stage of filling; A' value, atrial tissue velocity of the left atrioventricular valve annulus.

non-invasive Doppler ultrasound measurements as the indicator of LVD (13).

At present, the evaluation of LVD function is mainly based on left ventricular filling (hemodynamic changes), myocardial motion (particularly at the mitral annulus) and the structure of the left atrium and left ventricle (29). Doppler tissue imaging is able to reflect myocardial velocity in patients and the occurrence and cessation of the motion waveforms near the diastolic and systolic phases in the same cardiac cycle, based on which the LVD function of patients is scientifically evaluated (30).

LVD function is currently evaluated by the ratio of the early diastolic peak at the mitral valve (E) to the mitral annulus velocity (Em) measured by echocardiographic pulses and tissue Doppler imaging. An E/Em value of $>15$ suggests that the left ventricular filling pressure is elevated and an E/Em of $<8$ is considered normal (13). This indicator is not affected by ejection fraction, atrial fibrillation or sinus tachycardia and is rarely influenced by left ventricular filling pressure or the pressure gradient across the mitral valve. Ishikawa et al (31) reported LVD function and silent brain infarction in patients with non-valvular atrial fibrillation by tissue Doppler measurements. Olsen et al (32) recommend the use of tissue Doppler imaging to predict diastolic myocardial dysfunction in patients with CI. However, the feasibility of predicting acute CI by examining LVD function with tissue Doppler imaging in young hypertensive patients has not been previously reported, to the best of our knowledge. The present clinical study fills this research gap.

From the closure of the aortic valve, the LVD process may be divided into the isovolumetric relaxation period, the rapid filling period, the reduced filling period and the left atrial systolic period. The contribution rates of these different periods are 45-50, 35-40, 5 and 5-15\%, respectively (33). QTVI by tissue Doppler imaging may, in turn, reflect LVD function during the isovolumetric relaxation period, rapid filling period and left atrial systolic period by measuring IVRT, Ea/Aa and A' (34). The higher the IVRT and A'values and the lower the Ea/Aa, the more severe is the LVD function damage.

In the present study, the LVD function of CI patients prior to infarction was lower than that of patients post-infarction and the difference was statistically significant. The LVD function of CI patients post-infarction was lower than that of patients with non-CI patients and the difference was statistically significant. These data indicate that LVD function has a certain influence on the occurrence of CI in young hypertensive patients. It was indicated that the LVD function of patients with acute CI was lower than that of patients with hypertension alone and the difference was statistically significant. Conversely, the risk of acute CI is also significantly higher in young hypertensive patients with lower left ventricular function than in patients with normal left ventricular function. This difference in risk further indicates that early monitoring of left ventricular function has important clinical significance for young hypertensive patients to prevent acute CI. Furthermore, the probability of acute CI in young hypertensive patients with decreased left heart function is significantly elevated and the outcome indicated that the LVD function during the isovolumetric relaxation period and rapid filling period was severely impaired in patients with acute YIS. There are two probable reasons why impaired LVD function tends to occur during these two phases and trigger acute $\mathrm{CI}$ : i) In the majority of cases, CI occurs in these two phases; diastolic filling is obviously limited when damage is present and the blood volume return is reduced. In turn, the amount of blood ejection in the left ventricular systolic phase is insufficient and the peripheral circulatory blood volume is decreased, causing the cerebral perfusion blood volume to decline significantly. ii) The impairment of LVD function during these two phases results in reduced fluid shear stress on the vessel wall. In the long term, the conditions of reduced shear stress may induce and promote atherosclerosis. Furthermore, head or neck vascular atherosclerosis is well known as the most common cause of acute CI (35). Together, these two causes significantly increase the incidence of acute CI.

In conclusion, the present clinical study suggests that hypertension in young individuals is associated with decreased LVD function and is a risk factor for diastolic dysfunction in the left ventricle. An association exists between acute YIS and LVD dysfunction, which may help predict the onset of acute YIS. Hence, LVD functional measurement is relevant to the individualized treatment and prognostic evaluation of patients with YIS.

However, the present study had certain shortcomings. First, the association between hypertension grading and LVD function grading in young hypertensive patients remains to be fully established. In addition, the LVD function of young patients with acute CI is not regularly assessed. Furthermore, the effect of anti-hypertensive drugs on LVD and CI was not 
assessed in the present study, and this may be determined in future studies. Therefore, the dynamics of these indicators and associated changes were not observed. Finally, the literature on the association between LVD function classification and the CI area was sparse and further large-scale, multicenter clinical studies may be required to assess this.

\section{Acknowledgements}

The authors would like to thank Ms. Xiao-Di Fu (Department of Neurology, Baoji Municipal Central Hospital, China) for her technical assistance in drawing charts in the present study.

\section{Funding}

No funding was received.

\section{Availability of data and materials}

The datasets used and/or analyzed during the current study are available from the corresponding author on reasonable request.

\section{Authors' contributions}

HJW and XYW conceived, designed and drafted the manuscript, were responsible for the acquisition analysis, revision and interpretation of data and read and approved of the final version to be published. HJW and XYW agree to be accountable for all aspects of the work in ensuring that questions related to the accuracy or integrity of any part of the work.

\section{Ethics approval and consent to participate}

The present study was approved by the Ethics Committee of Baoji Municipal Central Hospital (Baoji, China) and the patients provided written informed consent.

\section{Patient consent for publication}

Written informed consents were obtained for publication.

\section{Competing interests}

The authors declare that they have no competing interests.

\section{References}

1. Heckman GA, Patterson CJ, Demers C, St Onge J, Turpie ID and McKelvie RS: Heart failure and cognitive impairment: Challenges and opportunities. Clin Interv Aging 2: 209-218, 2007.

2. Hooghiemstra AM, Bertens AS, Leeuwis AE, Bron EE, Bots ML, Brunner-La Rocca HP, de Craen AJ, van der Geest RJ, Greving JP, Kappelle LJ, et al: The missing link in the pathophysiology of vascular cognitive impairment: Design of the heart-brain study. Cerebrovasc Dis Extra 7: 140-152, 2017.

3. Banecka-Majkutewicz Z, Sawula W, Kadzinski L, Wegrzyn A and Banecki B: Homocysteine, heat shock proteins, genistein and vitamins in ischemic stroke-pathogenic and therapeutic implications. Acta Biochim Pol 59: 495-499, 2012.

4. Pierdomenico SD, Pierdomenico AM, Di Carlo S, Di Tommaso R and Cuccurullo F: Left atrial enlargement and risk of ischemic stroke in elderly treated hypertensive patients. Am J Hypertens 27: 1179-1184, 2014.
5. Nam KW, Kwon HM, Kim HL and Lee YS: Left ventricular ejection fraction is associated with small vessel disease in ischaemic stroke patients. Eur J Neurol 26: 747-753, 2019.

6. Cerrato P, Grasso M, Imperiale D, Priano L, Baima C, Giraudo M, Rizzuto A, Azzaro C, Lentini A and Bergamasco B: Stroke in young patients: Etiopathogenesis and risk factors in different age classes. Cerebrovasc Dis 18: 154-159, 2004.

7. Shimizu A, Kokubo M, Mitsui T, Miyagi M, Nomoto K, Murohara T, Toba K and Sakurai T: Left ventricular diastolic dysfunction is directly associated with cerebral white matter lesions in elderly patients. Geriatr Gerontol Int 15 (Suppl 1): S81-S82, 2015.

8. Ogata T, Matsuo R, Kiyuna F, Hata J, Ago T, Tsuboi Y, Kitazono $\mathrm{T}$ and Kamouchi M; FSR Investigators: Left atrial size and long-term risk of recurrent stroke after acute ischemic stroke in patients with nonvalvular atrial fibrillation. J Am Heart Assoc 6: e006402, 2017.

9. Smajlovic D: Strokes in young adults: Epidemiology and prevention. Vasc Health Risk Manag 11: 157-164, 2015.

10. Allaoui A, Echchilali K, Moudatir M, Alaoui FZ and Elkabli H: Causes of stroke among young people: Role of the internist. Pan Afr Med J 30: 114, 2018 (In French).

11. Kim SY, Song CM, Bang W, Lim JS, Park B and Choi HG: Nephrolithiasis predicts ischemic stroke: A longitudinal follow-up study using a national sample cohort. Int J Med Sci 16: 1050-1056, 2019.

12. Borlaug BA, Melenovsky V, Redfield MM, Kessler K, Chang HJ, Abraham TP and Kass DA: Impact of arterial load and loading sequence on left ventricular tissue velocities in humans. J Am Coll Cardiol 50: 1570-1577, 2007.

13. Manouras A, Nyktari E, Sahlen A, Winter R, Vardas P and Brodin LA: The value of E/Em ratio in the estimation of left ventricular filling pressures: Impact of acute load reduction: A comparative simultaneous echocardiographic and catheterization study. Int J Cardiol 166: 589-595, 2013.

14. Park CS, Kim YK, Song HC, Choi EJ, Ihm SH, Kim HY, Youn HJ and Seung KB: Effect of preload on left atrial function: Evaluated by tissue Doppler and strain imaging. Eur Heart J Cardiovasc Imaging 13: 938-947, 2012.

15. Rösner A, Avenarius D, Malm S, Iqbal A, Baltabaeva A, Sutherland GR, Bijnens B and Myrmel T: Persistent dysfunction of viable myocardium after revascularization in chronic ischaemic heart disease: Implications for dobutamine stress echocardiography with longitudinal systolic strain and strain rate measurements. Eur Heart J Cardiovasc Imaging 13: 745-755, 2012.

16. Chinese Medical Association C, CVD editorial board: Chinese heart failure diagnosis and treatment guide 2014. Chin J Cardiovascular Dis 42: 98-122, 2014.

17. Correale M, Totaro A, Ieva R, Ferraretti A, Musaico F and Di Biase M: Tissue Doppler imaging in coronary artery diseases and heart failure. Curr Cardiol Rev 8: 43-53, 2012.

18. Wang M, Hu B, Zhang YL, Shen E and Pan XQ: Effects of 3-aminobenzamide on ventricular function in infarct heart assessed by quantitative tissue velocity imaging. J Cardiovasc Med (Hagerstown) 17: 793-802, 2016.

19. Neurology branch of the Chinese Medical Association cvdg: Guidelines for the diagnosis and treatment of acute ischemic stroke in China 2014. Chin J Neurol 48: 246-257, 2015.

20. Gunasekaran P, Jeevanantham V, Sharma S, Thapa R and Gupta K: Implications of the 2013 ACC/AHA cholesterol guidelines on contemporary clinical practice for patients with atherosclerotic coronary and peripheral arterial disease. Indian Heart J 69: 464-468, 2017.

21. Bersano A, Borellini L, Motto C, Lanfranconi S, Pezzini A, Basilico P, Micieli G, Padovani A, Parati E and Candelise L: Molecular basis of young ischemic stroke. Curr Med Chem 20: 3818-3839, 2013.

22. Park TH, Ko Y, Lee SJ, Lee KB, Lee J, Han MK, Park JM, Cho YJ, Hong KS, Kim DH, et al: Identifying target risk factors using population attributable risks of ischemic stroke by age and sex. J Stroke 17: 302-311, 2015.

23. Cohen DL and Townsend RR: Approach to the young patient with new-onset hypertension. Clin J Am Soc Nephrol 13: 929-932, 2018.

24. Liu Q, Dong H, Meng L, Cheng H, Yan Y, Liu J and Mi J: Impacts of hypertension on early changes of cardiovascular structure and function among children: A case-control study. Zhonghua Liu Xing Bing Xue Za Zhi 36: 332-336, 2015 (In Chinese).

25. Alpsoy S, Oran M, Topcu B, Akyuz A, Akkoyun DC and Degirmenci H: Effect of lifestyle modifications on diastolic functions and aortic stiffness in prehypertensive subjects: A prospective cohort study. Anadolu Kardiyol Derg 13: 446-451, 2013. 
26. Seo JY, Lee KB, Lee JG, Kim JS, Roh H, Ahn MY, Park BW and Hyon MS: Implication of left ventricular diastolic dysfunction in cryptogenic ischemic stroke. Stroke 45: 2757-2761, 2014.

27. Gasiorek P, Sakowicz A, Banach M, von Haehling S and Bielecka-Dabrowa A: Arterial stiffness and indices of left ventricular diastolic dysfunction in patients with embolic stroke of undetermined etiology. Dis Markers 2019: 9636197, 2019.

28. Cherneva RV, Gospodinova MV, Denchev SV, Petkov RB, Kostadinov DE and Cherneva ZV: Stress echocardiography for left ventricular diastolic dysfunction detection in patients with non-severe chronic obstructive pulmonary disease: A cross-sectional study. Croat Med J 60: 449-457, 2019.

29. Schiebler ML, Bhalla S, Runo J, Jarjour N, Roldan A, Chesler N and François CJ: Magnetic resonance and computed tomography imaging of the structural and functional changes of pulmonary arterial hypertension. J Thorac Imaging 28: 178-193, 2013.

30. Zamojska J, Niewiadomska-Jarosik K, Wosiak A, Lipiec P and Stanczyk J: Myocardial dysfunction measured by tissue Doppler echocardiography in children with primary arterial hypertension. Kardiol Pol 73: 194-200, 2015.

31. Ishikawa S, Sugioka K, Sakamoto S, Fujita S, Ito A, Norioka N, Iwata S, Nakagawa M, Takagi M, Miki Y, et al: Relationship between tissue Doppler measurements of left ventricular diastolic function and silent brain infarction in patients with non-valvular atrial fibrillation. Eur Heart J Cardiovasc Imaging 18: 1245-1252, 2017.
32. Olsen FJ, Jørgensen PG, Møgelvang R, Jensen JS, Fritz-Hansen T, Bech J, Sivertsen J and Biering-Sørensen T: Diastolic myocardial dysfunction by tissue Doppler imaging predicts mortality in patients with cerebral infarction. Int J Cardiovasc Imaging 31: 1413-1422, 2015.

33. Afolabi-Brown OO, Lynn Morris D and Pressman GS: Systolic mitral valve opening and absent isovolumic relaxation: Unusual hemodynamics of severe mitral regurgitation. Echocardiography 31: E189-E190, 2014.

34. Krzesiak-Lodyga A and Cwetsch A: Echocardiographic methods for assessment of left ventricular diastolic dysfunction-state of the art. Pol Merkur Lekarski 35: 63-66, 2013 (In Polish).

35. Nacu A, Fromm A, Sand KM, Waje-Andreassen U, Thomassen L and Naess H: Age dependency of ischaemic stroke subtypes and vascular risk factors in western Norway: The Bergen Norwegian stroke cooperation study. Acta Neurol Scand 133: 202-207, 2016. 